\title{
Truncated Balitskiı-Fadin-Kuraev-Lipatov series and hadronic collisions
}

\author{
M. B. Gay Ducati* and M. V. T. Machado ${ }^{\dagger}$ \\ Instituto de Física, Universidade Federal do Rio Grande do Sul, Caixa Postal 15051, CEP 91501-970, Porto Alegre, RS, Brazil
}

(Received 22 August 2000; revised manuscript received 27 November 2000; published 9 April 2001)

\begin{abstract}
We study the contribution of a truncated Balitski1-Fadin-Kuraev-Lipatov Pomeron series to the hadronic processes showing that a reliable description is obtained using two orders in perturbation theory. The $p p(p \bar{p})$ total cross sections are described with good agreement, consistent with the unitarity bound. We also calculate the elastic-scattering amplitude at nonzero momentum transfer $t$, introducing two distinct Ansätze for the proton impact factor. As a by product the elastic differential cross section is obtained at the small $t$ approximation and compared with the data, describing with good agreement this observable for both low- and highenergy values.
\end{abstract}

DOI: 10.1103/PhysRevD.63.094018

PACS number(s): 12.38.Bx, 13.60.Hb

\section{INTRODUCTION}

Several years ago the calculation program of the perturbative contribution to the Balitskii-Fadin-Kuraev-Lipatov (BFKL) Pomeron was started, generating the integral equation that determines its behavior in perturbative QCD [1]. That procedure consists of summing the leading logarithms on energy [leading log approximation (LLA)], $\ln (s)$, order by order from perturbation theory, selecting those sets of Feynman diagrams corresponding to ladders. In the LLA, such diagrams are constructed with Reggeized gluons in the $t$ channel and bare gluons in the $s$ channel (the rungs), which are connected by a nonlocal gauge-invariant effective vertex. The resultant physical picture is that the color singlet exchange is associated with a gluon ladder with infinite rungs [1]. The main result is that the total cross section for the exchange process is a power of the center-of-mass energy, which leads to the mathematical definition of the BFKL Pomeron as a cut rather than a pole in the complex angular momentum plane [2].

Such behavior is inconsistent with the requirement of the unitarity bound [3] and a unitarization procedure has to be performed. The unitarity constraint states that the total cross section may not grow faster than $\ln ^{2}(s)$. Therefore, corrections in order to avoid unitarity violation present in the amplitude (i.e., total cross section) in the BFKL approach should be taken into account. In the BFKL approach the violation of unitarity is due to the fact that the $s$-channel cut amplitudes contain only a subset of all the possible intermediate states, namely, only gluons in the leading logarithmic approximation and gluons plus a $q \bar{q}$ pair in the next-leading approximation (NLLA). Therefore, we are unable to restore unitarity in the BFKL approach even in the NLLA. In Ref. [4] the restoration is based on using unitarity and dispersion relations from the start as a tool to construct higher-order amplitudes. The main result of this approach is the need to take into account contributions with higher number of Reggeized gluons in the $t$ channel, compared to the BFKL

\footnotetext{
*Email address: gay@if.ufrgs.br

†Email address: magnus@if.ufrgs.br
}

amplitude with two Reggeized gluons. In Ref. [5], the unitarity problem could be solved by resumming all multiple BFKL Pomeron (at LLA) exchanges in the total cross section. Despite the intense theoretical work at present, the unitarization problem still remains an open question.

A priori, BFKL is itself asymptotic and we may ask if at finite energies, i.e., nonasymptotic regime, a finite sum of the BFKL series could describe the existent data. Recently, Fiore et al. [6] performed a reasonable fit to data on $p p(p \bar{p})$ total cross section using this hypothesis. They considered the $n$-rung ladder diagrams, with $n=0,1,2$, and 3 , at distinct energy intervals and the parameters are fitted for each interval. Such a procedure introduces a large set of parameters. An additional fact is that contributions from subleading diagrams in the perturbative expansion are absorbed into the parameters. These features turn the analysis involved when one considers unitarization or calculation of nonforward observables, as the elastic differential cross section.

A well-known property of perturbative QCD calculations is that there are several reasons to believe that the region $t$ $\rightarrow 0$ plays a very special role and perturbation theory may even not be applicable. Although this is a fact, in the recent literature, the forward region in hadronic collisions is treated based on the scale anomaly of QCD, maintaining a perturbative approach supported by a large scale from the QCD vacuum [7], obtaining consistent results with those ascribed phenomenologically to the soft Pomeron. In our case, despite the restrictions imposed by the use of a perturbative description for soft observables, there is sufficient motivation to perform a deeper analysis on the BFKL series. In order to make this we should use the set of diagrams producing contributions $\sim\left[\alpha_{s} \ln (s)\right]^{n}$, order by order from perturbation theory, performing a finite sum of gluon ladders. We notice that when one refers to ladders we have in mind that they are constructed by Reggeized gluons and effective vertices. The question that remains is how many orders to take into account? The lowest-order two gluons exchange calculation leads to a total cross section constant on energy. This is a crude approximation to the reality, since experimentally the cross section has a slow increase with the energy and therefore higher-order contributions are necessary.

The next contribution to the sum is the one rung gluon 
ladder. This calculation provides a logarithmic growth of the total cross section on energy, scaled by the typical gluon transverse momentum (in LLA it is arbitrary). In order to avoid unitarity violation and by simplicity we truncate our summation at this order, supported by the knowledge that a logarithm behavior is in agreement with the experimental results from a dispersion relation fit [8]. In our case the selected diagrams cover all energy ranges, instead of defining a distinct set of diagrams for subintervals of energy as in Ref. [6]. As a result we performed a successful fit to the protonproton(antiproton) total cross sections with these two contributions. These results motivate us to check the nonforward amplitude in order to obtain the prediction for the elastic differential cross section, which gives the behavior on the momentum transfer $t$.

In the BFKL framework such analysis is dependent on both the proton impact factor input and the Lipatov kernel. The BFKL kernel, i.e., Green's function for the Reggeized gluons, is not physical but is under control since it is calculated from perturbative QCD. For example, the cancellation of the infrared singularities in the kernel is known from Ref. [9]. The amplitude describing the interaction of the particles (colorless) is the convolution of the kernel with the corresponding impact factors and it should be infrared safe. In our case, the main uncertainty arises from modeling the proton impact factor, which presents nonperturbative content. The impact factors determine the coupling of the Pomeron to the color singlet hadrons and necessarily vanish when the transverse momentum of any gluon vanishes, which is required for the cross section to be finite. The infrared singularities cancellation in the impact factor of colorless particles has been demonstrated to next-to-leading order in Ref. [10]. Moreover, the impact factor plays a crucial rule in the calculation of the nonforward amplitude, in fact determining its $t$ dependence.

We calculate the proton-proton(antiproton) elasticscattering amplitude at nonzero momentum transfer $t$ taking into account two distinct Ansätze to the proton impact factor, the Dirac form factor, which has explicit $t$ dependence and is decoupled in the gluon transverse momenta, as proposed recently by Balitskiı and Kuchina [11]. The calculation was also performed with an usual impact factor [12], whose shape is determined by quite general properties and was considered for comparison. The main resulting features are discussed, having in mind that a more realistic ansatz to the proton impact factor is still to be found.

This paper is organized as follows. In the next section we present a short review of the formulas concerning the two gluons exchange and one rung contribution to the BFKL approach, presenting the details of the fit to the protonproton (antiproton) total cross sections. In Sec. III, one presents the results to the nonforward elastic-scattering amplitude with two distinct impact factor models and their main features are discussed. The elastic differential cross section is calculated in the small $t$ approximation and compared with the experimental data at two distinct energy regimes. In the last section we present our conclusions.

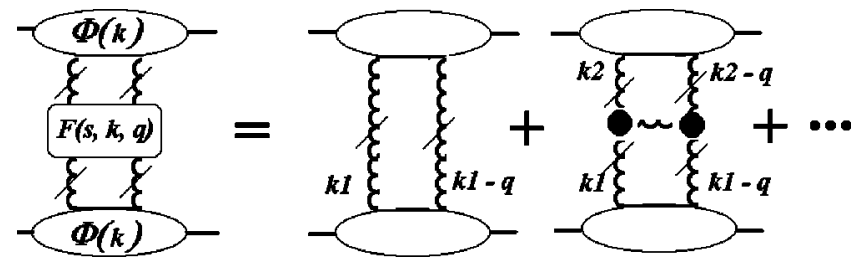

FIG. 1. The blobs denote the proton (antiproton) structure (impact factors) and the first two orders in perturbation theory are shown. In LLA, the ladder is constructed with Reggeized gluons in the $t$ channel and bare gluons on the $s$ channel (the rungs), which are connected by a nonlocal gauge-invariant effective vertex (the bold blob).

\section{THE TRUNCATED BFKL SERIES}

By calculating order by order in perturbation theory, summing over the leading logarithms of the center-of-mass energy $s$, one obtains the BFKL equation, which describes the scattering process by an infinite rung gluon ladder exchange (see Fig. 1). In this approach, called leading logarithm approximation, the Pomeron is obtained considering the color singlet ladder diagrams whose vertical lines are Reggeized gluons coupled to the rungs (bare gluons) through the effective vertices. The correspondent amplitude is purely imaginary and the coupling constant $\alpha_{s}$ is considered frozen in some transverse momentum scale.

For the elastic scattering of a hadron, the Mellin transform of the scattering amplitude is given by [13]

$$
\mathcal{A}(\omega, t)=\frac{\mathcal{G}}{(2 \pi)^{2}} \int d^{2} \mathbf{k}_{1} d^{2} \mathbf{k}_{2} \frac{\Phi\left(\mathbf{k}_{1}\right) \Phi\left(\mathbf{k}_{2}\right)}{\mathbf{k}_{2}^{2}\left(\mathbf{k}_{1}-\mathbf{q}\right)^{2}} f\left(\omega, \mathbf{k}_{1}, \mathbf{k}_{2}, \mathbf{q}\right),
$$

where the $\mathcal{G}$ is the color factor for the color singlet exchange, $\mathbf{k}_{1}$ and $\mathbf{k}_{2}$ are the transverse momenta of the exchanged gluons in the $t$ channel, and $\mathbf{q}$ is the momentum transfer, with $\mathbf{q}^{2}=-t$. The impact factors describing the interacting particles transition in the particle-Reggeon (i.e., the Reggeized gluons) processes are by definition factorized from the Mellin transform of the Green's function for the ReggeonReggeon scattering. As a consequence, the energy dependence is determined by the function $f\left(\omega, \mathbf{k}_{1}, \mathbf{k}_{2}, \mathbf{q}\right)$. This fact turns evident once one defines the transform

$$
f(\omega)=\int_{1}^{\infty} d\left(\frac{s}{\mathbf{k}^{2}}\right)\left(\frac{s}{\mathbf{k}^{2}}\right)^{-\omega-1} F(s) .
$$

In Eq. (2), a function that is a pure power of $s$ produces a simple pole on $\omega$; otherwise, as a power of $\ln s$ the transform has a cut singularity. Therefore the $s$ dependence of the amplitude is obtained from the singularity structure of the transforms.

The function $f\left(\omega, \mathbf{k}_{1}, \mathbf{k}_{2}, \mathbf{q}\right)$ is the Mellin transform of the BFKL kernel $F\left(s, \mathbf{k}_{1}, \mathbf{k}_{2}, \mathbf{q}\right)$, which states the dynamics of the process and is completely determined in perturbative QCD. The main properties of the LO kernel are well known [1] and the results arising from the NLO calculations have yielded intense debate in the literature recently [14]. 
In the case of $p p(p \bar{p})$ scattering, the factor $\Phi(\mathbf{k})$ is the proton impact factor, which in the absence of a perturbative scale has a nonperturbative feature and provides the Pomeron-proton coupling. This factor avoids the infrared divergences arising from the transverse momentum integration. However, it introduces some uncertainty in the amplitude calculation since it is not obtained from QCD first principles.

In the leading order of perturbation theory we have

$$
f_{1}\left(\omega, \mathbf{k}_{1}, \mathbf{k}_{2}, \mathbf{q}\right)=\frac{1}{\omega} \delta^{2}\left(\mathbf{k}_{1}-\mathbf{k}_{2}\right),
$$

and in the next order

$$
\begin{aligned}
f_{2}\left(\omega, \mathbf{k}_{1}, \mathbf{k}_{2}, \mathbf{q}\right)= & -\frac{\bar{\alpha}_{s}}{2 \pi} \frac{1}{\omega^{2}}\left[\frac{\mathbf{q}^{2}}{\mathbf{k}_{1}^{2}\left(\mathbf{k}_{2}-\mathbf{q}\right)^{2}}\right. \\
& \left.-\frac{1}{2} \frac{1}{\left(\mathbf{k}_{1}-\mathbf{k}_{2}\right)^{2}}\left(1+\frac{\mathbf{k}_{2}^{2}\left(\mathbf{k}_{1}-\mathbf{q}\right)^{2}}{\mathbf{k}_{1}^{2}\left(\mathbf{k}_{2}-\mathbf{q}\right)^{2}}\right)\right] .
\end{aligned}
$$

For convenience we define $\bar{\alpha}_{s}=N_{c} \alpha_{s} / \pi$, where $N_{c}$ is the color number and $\alpha_{s}$ is the strong-coupling constant fixed at transverse momentum scale. In order to perform a reliable calculation the convenient proton impact factor should be introduced. This is not an easy task, namely, these hadronic processes are soft and there is no hard scale allowing us to use the perturbation theory. In fact, we should know in detail the parton wave function in the hadron to calculate the impact factors properly. Since this is not available, several models are proposed in order to calculate them. This problem is addressed in the next section.

Now we study the results produced when one considers a truncation of the full BFKL series at the forward (or near forward) region, i.e., $t=0$. The scattering amplitude, Eq. (1), can be used to calculate the $p p(p \bar{p})$ total cross sections. From the optical theorem the relation between the total cross section and the scattering amplitude is $\sigma_{\text {tot }}=\Im m \mathcal{A}(s, t$ $=0) / s$, having the lowest-order contribution (Born level) as a constant term in energy, and the next order term as a logarithm, scaled by a typical gluon transverse momentum of the process (bearing in mind it is arbitrary). When considering zero-momentum-transfer there is no need to deal with both a specific form for the impact factor and the transverse momentum integration. This allows to consider $s$-independent factors in each term as free parameters and to obtain them from data.

We select the set of data on proton-proton(antiproton) total cross section [15], considering points with $\sqrt{s}>4 \mathrm{GeV}$ to avoid very low-energy data, and choose the typical transverse momentum as $\mathbf{k}^{2}=s_{0}=1 \mathrm{GeV}^{2}$, in such a way that the factors are in $(\mathrm{mb})$. The correct description at low energy requires the Reggeon contribution, which is parametrized from Regge theory. Our expression to the total cross section is then,

$$
\sigma_{\text {tot }}^{p p(p \bar{p})}=C_{R}\left(s / s_{0}\right)^{\alpha_{R}(0)-1}+C_{B o r n}+C_{N O} \ln \left(s / s_{0}\right) .
$$

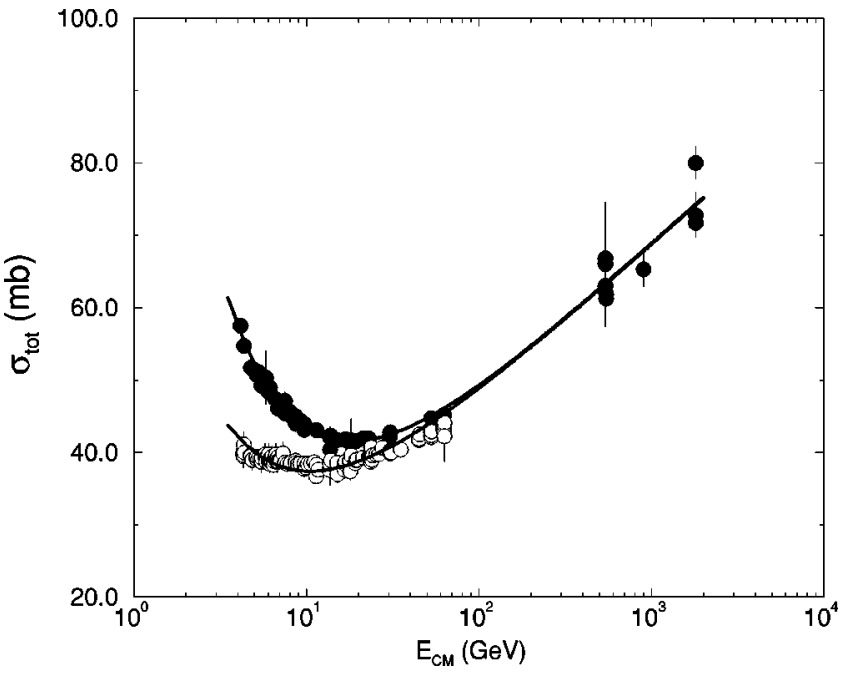

FIG. 2. Result of the $p p(p \bar{p})$ total cross sections, Ref. [15]. The errors are added into quadrature.

The Reggeon intercept at zero-momentum transfer is $\alpha_{R}(0)$ and the factor $C_{R}$ is distinct to $p p$ and $p \bar{p}$, as a consequence of the different Reggeon coupling to particle and antiparticle. Consistent with the usual Donnachie-Landshoff fit [16] for $p \bar{p}$, the Reggeon contribution is described effectively by $\alpha_{R}=0.5475$. Hence we fix the constants $C_{B o r n}$ and $C_{N O}$ from data on $p \bar{p}$, imposing the same contribution for both proton-proton and proton-antiproton. This procedure is reasonable due to the higher energies reached on $p \bar{p}$ collision, where the Pomeron dominates. On the other hand, $p p$ data are predominantly at low energy, which is not strongly sensitive to the Pomeron model, thus dominated by the Reggeonic contribution. In the $p p$ case there is need of a more refined parametrization for the Reggeonic piece [16], therefore we consider the intercept as a free parameter for this process. A successful description of data is obtained for the whole range of energy. The result is shown in Fig. 2, and the parameters are presented in Table I.

Our result is similar to that of Fiore et al. [6], with the advantage to use a smaller set of parameters (see Table I), and no additional soft Pomeron is used in our analysis. We use only two orders in perturbation theory (up to the one rung ladder) while the authors in Ref. [6] use up to the fourth order. We describe the $p \bar{p}$ total cross section with only three free parameters (five for $p p-p \bar{p}$ simultaneous fit), instead of 12 or 16 (considering up to two rungs or three rungs ladder, respectively) from Ref. [6]. An important additional

TABLE I. The parameters for $p \bar{p}(p p)$ cross sections $\left(\chi^{2}\right.$ $=1.19$ ).

\begin{tabular}{lrlcc}
\hline \hline Process & $C_{R}$ & $\alpha_{R}(0)$ & $C_{\text {Low/Nuss }}$ & $C_{\text {rung }}$ \\
\hline$p \bar{p}$ & 141.51 & 0.5475 & 4.16 & 4.66 \\
$p p$ & 78.15 & 0.589 & 4.16 & 4.66 \\
\hline \hline
\end{tabular}


advantage is that the total cross section obtained is consistent with the unitarity constraint, avoiding eikonalization procedures.

The hypothesis of considering two orders from the BFKL series, namely, $\sigma_{\text {tot }} \sim \ln (s)$, is phenomenologically corroborated by the well-known dispersion relation fit [8]. This fit is based on measurements of $\sigma_{\text {tot }}$ and $\rho$ parameter in energies $5 \mathrm{GeV} \leqslant \sqrt{s} \leqslant 546 \mathrm{GeV}$ and the high-energy dependence was described by $\sim\left[\log \left(s / s_{0}\right)\right]^{\gamma}$, with $\gamma=2.2 \pm 0.3$. A simple logarithmic behavior, $\gamma=1$, is favored by the results of the experimental group E710/E811 [17] at $\sqrt{s}=1800 \mathrm{GeV}$ and supported by the very high-energy cosmic ray data [18]. As a final remark, at the CERN Large Hadron Collider energy $(\sqrt{s}=14 \mathrm{TeV})$ the extrapolation of our results will give $\sigma_{\text {tot }}=93.22 \mathrm{mb}$.

In the next section we calculate the elastic amplitude at nonzero momentum transfer using two distinct models for the proton impact factor, discussing its main properties, and obtaining a description of the existent data in the small- $t$ approximation.

\section{THE NONFORWARD-SCATTERING AMPLITUDE}

Now we perform an analysis of the elastic-scattering amplitude at nonzero-momentum transfer $t=-\mathbf{q}^{2}$. In order to calculate this amplitude, information about the coupling between the proton and the $t$-channel gluons in the ladder is required. Namely, we should introduce a reliable proton impact factor.

In the calculation of the hadron-hadron scattering amplitude the basic diagram is the quark-quark elastic scattering, which are taken on shell. This fact does not correspond to reality since the Pomeron couples to the hadron whose constituent partons are slightly off shell. For the quark-quark case, although $f\left(\omega, \mathbf{k}_{1}, \mathbf{k}_{2}, \mathbf{q}\right)$ does not contain any infrared singularities, the amplitude nevertheless diverges due to the remaining integrals over $\mathbf{k}_{1}$ and $\mathbf{k}_{2}$, which develop infrared singularities when $\mathbf{k}_{1}$ and $\mathbf{k}_{2}$ [or $\left(\mathbf{k}_{1}-\mathbf{q}\right),\left(\mathbf{k}_{2}-\mathbf{q}\right)$ ] go to zero. In principle, when we convolute the bare amplitude with the impact factors it should be infrared safe. The next task is to model the impact factor since it cannot be calculated from first principles due to the unknowledge on the wave function of the hadronic constituent partons.

Here are analyzed two distinct models for the impact factor and its consequences for the elastic amplitude and the differential cross section.

\section{A. Dirac form factor}

Balitskiı and Kuchina proposed recently [11] that at large momentum transfer the coupling of the BFKL Pomeron to the nucleon is essentially equal to the Dirac form factor of the nucleon. Their basic idea is that in the lowest order in perturbation theory there is no difference between the diagrams for the nucleon impact factor and similar diagrams with two gluons replaced by two photons, in such a way that the amplitudes can be calculated without any model assumption.
This impact factor is decoupled in the transverse momentum integration and presents an explicit dependence on $t$, being similar to the usual Pomeron-proton coupling used in Regge phenomenology. The expression is

$$
\Phi_{p}(\mathbf{k}, \mathbf{q})=F_{1}^{p+n}(t)=\frac{1}{1+\left(|t| / 0.71 \mathrm{GeV}^{2}\right)^{2}} \frac{4 m_{p}^{2}+0.88|t|}{4 m_{p}^{2}+|t|}
$$

The choice for this proton impact factor is useful when one analyzes near forward observables, for instance the elastic differential cross section. However, it does not play the role of a regulator of infrared divergencies arising from the calculations at proton-proton (antiproton) process, because clearly it does not vanish when the gluon transverse momenta goes to zero. In electron-proton process the situation is different since the photon impact factor supplies that condition [11].

Then the next step is to perform the gluon transverse momenta integrations. In fact, such integrals are infrared divergent and should be regularized. An usual way out is to introduce an infrared cutoff $\lambda^{2}$ (for instance, Ref. [19]), temporally defining a small gluon mass, avoiding problems at the infrared region. This procedure is quite similar as to take into account a nonperturbative massive gluon propagator (i.e., see Ref. [20]).

The lowest-order (order $\alpha_{s}^{2}$ ) contribution, i.e., the Pomeron at the Born level, gives the following result:

$$
\begin{aligned}
\mathcal{A}^{(1)}\left(s, t ; \lambda^{2}\right) & =\frac{\mathcal{G}^{\prime}}{(2 \pi)^{4}} s \int d^{2} \mathbf{k} \frac{\Phi_{p}^{2}(\mathbf{k})}{\mathbf{k}^{2}(\mathbf{k}-\mathbf{q})^{2}} \\
& =\frac{\mathcal{G}^{\prime}}{(2 \pi)^{4}} s\left[F_{1}^{p+n}(t)\right]^{2} \frac{\pi}{\left(|t|-\lambda^{2}\right)} \ln \left(\frac{\lambda^{2}}{|t|}\right) .
\end{aligned}
$$

Here we notice that there is an implicit dependence on $\lambda^{2}$ in the above equation. The one rung gluon ladder has two components (order $\alpha_{s}^{3}$ ), given by the following expression:

$$
\mathcal{A}^{(2)}\left(s, t ; \lambda^{2}\right)=\frac{\mathcal{G}^{\prime}}{(2 \pi)^{4}} s\left[F_{1}^{p+n}(t)\right]^{2} \ln \left(\frac{s}{\mathbf{k}^{2}}\right)\left(I_{1}+I_{2}\right),
$$

with $I_{1}$ corresponding to the one rung gluon ladder and $I_{2}$ corresponding to the three gluons exchange graphs, whose order is also $\ln \left(s / \mathbf{k}^{2}\right)$. Such structure is due to the fact that in the color singlet calculation there is no cancellation between graphs and one cannot obtain an expression for the two-loop level, which is proportional to the one-loop amplitude [13]. We define $I_{2}$ through symmetry on the integration variables $\mathbf{k}_{1}$ and $\mathbf{k}_{2}$ [see Eqs. (1)-(4)] and the factor $\mathcal{G}^{\prime}$ collects the correspondent color factors and the remaining constants. The explicit calculation of those integrals, yields 


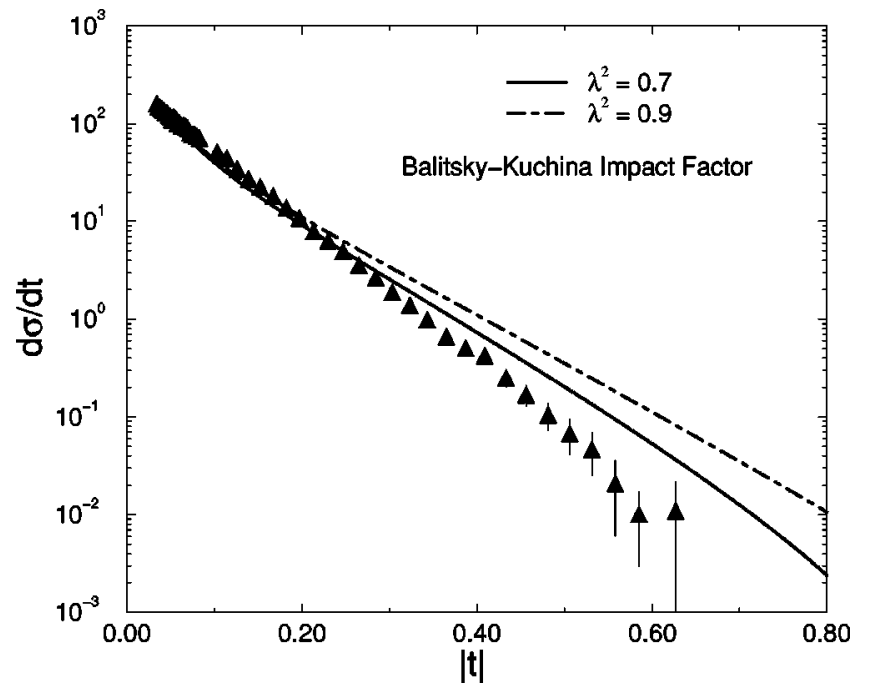

FIG. 3. The predicted differential cross section using the Balitski1-Kuchina impact factor, Ref. [11], and the experimental results at $1800 \mathrm{GeV}$, for two distinct values of the cutoff $\lambda^{2}$.

$$
\begin{aligned}
I_{1} & =-\mathbf{q}^{2} \int d^{2} \mathbf{k}_{1} \frac{1}{\mathbf{k}_{1}^{2}\left(\mathbf{k}_{1}-\mathbf{q}\right)^{2}} \int d^{2} \mathbf{k}_{2} \frac{1}{\mathbf{k}_{2}^{2}\left(\mathbf{k}_{2}-\mathbf{q}\right)^{2}} \\
& =-\pi^{2} \frac{|t|}{\left(|t|-\lambda^{2}\right)^{2}} \ln ^{2}\left(\frac{\lambda^{2}}{|t|}\right) \\
I_{2} & =\int d^{2} \mathbf{k}_{1} \int d^{2} \mathbf{k}_{2} \frac{1}{\mathbf{k}_{1}^{2}\left(\mathbf{k}_{1}-\mathbf{k}_{2}\right)^{2}\left(\mathbf{k}_{2}-\mathbf{q}\right)^{2}} \\
& =\frac{1}{2} \frac{\pi^{2} \ln \left(\lambda^{2}\right)}{\left(|t|-\lambda^{2}\right)} \ln \left(\frac{\lambda^{2}}{|t|}\right)\left(1-\frac{\ln (|t|)}{\ln \left(\lambda^{2}\right)}\right)
\end{aligned}
$$

Some comments about the amplitude above are in order. The scale for the factor $\lambda^{2}$ should be at nonperturbative regime, i.e., $\lesssim 1 \mathrm{GeV}^{2}$. In Fig. 3 we show a comparison between the predicted differential cross section using the Balitskii-Kuchina impact factor and the experimental results at $1800 \mathrm{GeV}$. An analysis is performed for two distinct values of the cutoff $\lambda^{2}$. The prediction presents a deviation of the usual exponential parametrization from Regge phenomenology and a remarkable difference appears at larger $t$ values. In addition, the impact factor $\Phi_{p}(\mathbf{k}, \mathbf{q})$ above does not satisfy the condition $\Phi(\mathbf{k}=0, \mathbf{q})=\Phi(\mathbf{k}=\mathbf{q}, \mathbf{q})=0$, required for the corresponding cross section to be finite [10], giving rise to the singularity at $t=0$ for the calculated amplitude.

Moreover, an interesting aspect is the behavior of the amplitude at the forward limit $t=0$, where it became very large. This limit is a well-known property of perturbative QCD calculations and there are several reasons to believe that the point $t=0$ plays a very special role, such that perturbation theory may not even be applicable. Concerning the forward region, for the full BFKL series, there is still the diffusion on transverse momenta, i.e., on $\ln \mathbf{k}^{2}$, which extends into both the ultraviolet and the infrared regions [21]. Nevertheless, the momentum scale $t$ supplies the control condition.
However, we suppose that a smooth transition from a finite $t$ down to $t=0$ is possible and that the truncated BFKL series gives the correct behavior on energy for the forward observables. Later we make use of this hypothesis to get a parametrization to the logarithmic slope $B(s)$ and calculate the differential elastic cross section.

\section{B. Usual nonperturbative ansatz}

Using quite general properties of the impact factors, namely, they vanish as the $t$-channel gluons transverse momenta go to zero, one can guess their behavior, which is determined by the large-scale nucleon dynamics. Such study has been performed at Ref. [12], where the solutions of the Lipatov equation are examined critically and their importance on the structure function description determined using physically motivated modifications for small $\mathbf{k}^{2}$. Namely, it performed a detailed parametrization of the infrared region that satisfies the gauge invariance constraints when $\mathbf{k}^{2} \rightarrow 0$. We use this result to study its role in our calculation for the elastic amplitude. The impact factor is written now as

$$
\Phi_{p}(\mathbf{k})=\frac{\mathbf{k}^{2}}{\mathbf{k}^{2}+\mu^{2}},
$$

where $\mu^{2}$ is a scale that is typical of the nonperturbative dynamics and is related to the radius of the gluonic form factor of the proton. Considering it as the scale of the hadronic electromagnetic form factor, then $\mathbf{k}^{2} \simeq 0.5 \mathrm{GeV}^{2}$ instead of estimates from QCD sum rules giving $\mathbf{k}^{2}$ $\simeq 1-2 \mathrm{GeV}^{2}[12]$.

As a consequence of this choice for the impact factor, the momentum-transfer behavior is completely determined by the kernel, since we consider $\mathbf{q} \neq 0$. The amplitude now reads

$$
\begin{aligned}
\mathcal{A}(s, t)= & \frac{\mathcal{G}}{(2 \pi)^{4}} s \pi I_{1}\left(t, \mu^{2}\right)+\frac{\mathcal{G}}{(2 \pi)^{4}} s \pi \ln \left(\frac{s}{\mathbf{k}^{2}}\right) \\
& \times\left[I_{1}^{2}\left(t, \mu^{2}\right)+I_{2}\left(t, \mu^{2}\right)\right],
\end{aligned}
$$

where

$$
\begin{aligned}
& I_{1}\left(t, \mu^{2}\right)=\frac{1}{\left(|t|-\mu^{2}\right)}+\frac{|t|}{\left(|t|-\mu^{2}\right)^{2}} \ln \left(\frac{\mu^{2}}{|t|}\right), \\
& I_{2}\left(t, \mu^{2}\right)=\frac{\ln \left(\mu^{2}\right)}{\left(|t|-\mu^{2}\right)}+\frac{\ln \left(\mu^{2}\right)|t|}{\left(|t|-\mu^{2}\right)^{2}} \ln \left(\frac{\mu^{2}}{|t|}\right) .
\end{aligned}
$$

In Fig. 4 we present the prediction to the differential cross section at $1800 \mathrm{GeV}$, using two distinct values for the parameter $\mu^{2}$. Again a deviation from the exponential parametrization based on Regge phenomenology is present, mainly at larger $t$. We observe again a divergent behavior at $t=0$, as a consequence of the impact factor, which does not satisfy the condition $\Phi(\mathbf{k}=0, \mathbf{q})=\Phi(\mathbf{k}=\mathbf{q}, \mathbf{q})=0$.

Despite obtaining an analytic expression to the elasticscattering amplitude, i.e., differential cross section, a direct comparison with the whole experimental data is known not 


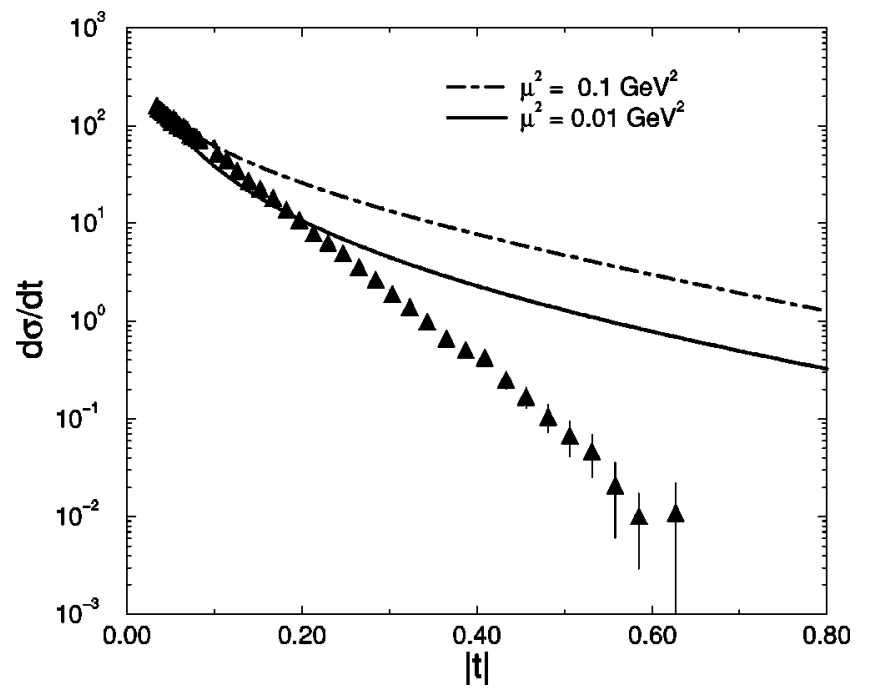

FIG. 4. The prediction to the differential cross section using the usual impact factor, Ref. [12], and the experimental results at 1800 $\mathrm{GeV}$, Ref. [15], using two distinct values for the parameter $\mu^{2}$.

to be reliable. To perform a more phenomenological analysis we notice that one can parametrize the elastic slope claiming that the forward amplitude is finite in $t=0$ and the dependence on energy is correctly described by the truncated BFKL series. Such an hypothesis is supported by the factorization on energy and momentum transfer present in the amplitudes. In addition, data on differential cross section at low $t$ are parametrized in the form $d \sigma / d t=A e^{B t}$, where $B$ is the forward slope [22]. Therefore, we can obtain an expression for the differential cross section at small $t$, using our previous results.

The usual relation to describe the cross section is

$$
\begin{gathered}
\frac{d \sigma^{e l}}{d t}=\left.\frac{d \sigma}{d t}\right|_{t=0} e^{B(s, t=0) t}=\frac{\sigma_{t o t}^{2}}{16 \pi} e^{B_{e l}(s) t}, \\
B(s, t=0)=\frac{d}{d t}\left[\log \frac{d \sigma}{d t}\right] .
\end{gathered}
$$

In the Regge framework the $B$ slope is obtained from the powerlike behavior of the scattering amplitude, dependent on the effective slope of the Pomeron trajectory $\alpha_{P}^{\prime}$, namely, $B_{e l}^{R e g g e}(s)=4 b_{0}+2 \alpha_{P}^{\prime} \ln (s)$. The $b_{0}$ comes from the exponential parametrization for the slope of the proton-protonPomeron vertex. In our case we should calculate the slope from the nonforward elastic-scattering amplitudes $\mathcal{A}^{\text {Ladder }}(s, t)$ obtained above. For the amplitude obtained employing the Balitskiı and Kuchina impact factor, one obtains the following slope:

$$
B(s)=\left.\frac{4}{F_{1}^{p+n}(t)} \frac{d F_{1}^{p+n}(t)}{d t}\right|_{t=0}+\left.\frac{2}{\mathcal{A}^{\text {Ladder }}} \frac{d \mathcal{A}^{\text {Ladder }}}{d t}\right|_{t=0},
$$

where the first term does not contribute effectively at $t=0$ and we are left only with the second term. From simple inspection of the amplitude obtained with the usual impact

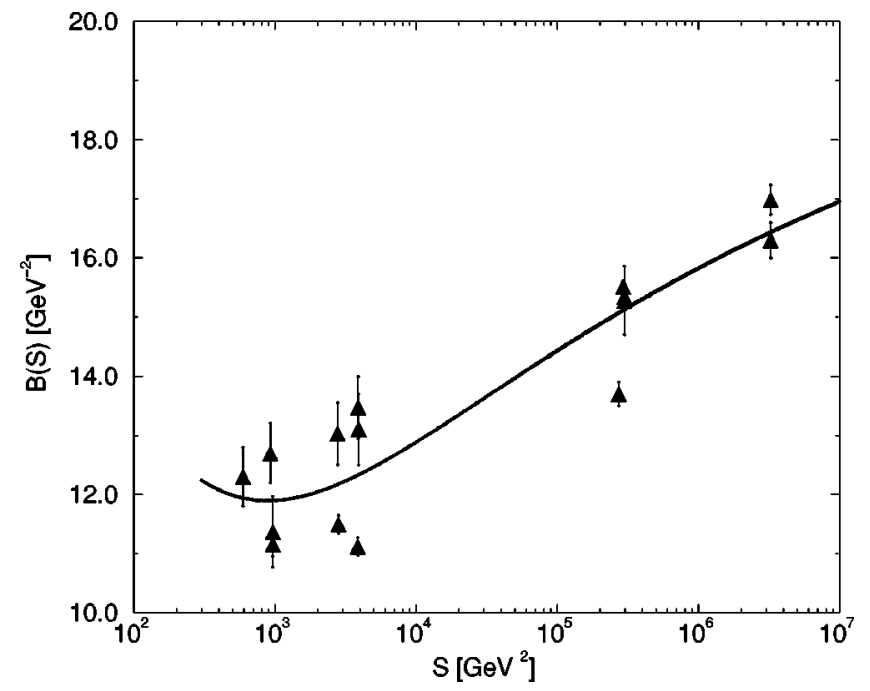

FIG. 5. The result for the slope $B(s)$, using both low- and highenergy data points on $p \bar{p}$ reaction, Ref. [15].

factor [see Eq. (12)] we also verify that one gets a similar expression to the correspondent slope.

Considering the specific form for the $t$ derivative of the amplitudes, their asymptotic values at $t=0$ depend only on the energy. In fact, they take the form $d \mathcal{A} / d t=R_{1} s$ $+R_{2} s \ln \left(s / s_{0}\right)$, where $R_{1}$ and $R_{2}$ are $s$-independent parameters. For our case, the amplitude is purely imaginary, then $|\mathcal{A}(s, t=0)|=s \sigma_{\text {tot }}$ and $d \sigma /\left.d t\right|_{t=0}=\sigma_{\text {tot }}^{2} / 16 \pi$. Putting all together, the corresponding slope and the elastic differential cross section are

$$
\begin{aligned}
B(s) & =\frac{2}{\sigma_{\text {tot }}}\left[R_{1}+R_{2} \ln \left(s / s_{0}\right)\right], \\
\frac{d \sigma}{d t} & =\frac{\sigma_{t o t}^{2}}{16 \pi} e^{B(s) t},
\end{aligned}
$$

where again $s_{0}=1 \mathrm{GeV}^{2}$.

In order to obtain the parameters $R_{1}$ and $R_{2}$, we use the slope experimental values for both low- [CERN Intersecting Storage Rings] and high-energy [CERN Super Proton Synchrotron, Fermilab Tevatron] points from $p \bar{p}$ reaction (23 $<\sqrt{s}<1800 \mathrm{GeV}$ ) [15]. The total cross section is given by Eq. (5). Our result is shown in Fig. 5, and the parameters are presented in Table II. For completeness we include the reggeon contribution since we also deal with low-energy data, requiring one additional parameter $\left(b_{R}\right)$ coming from the parametrization to the proton-proton-Reggeon vertex.

Having the slope obtained from data, the elastic differential cross section is straightforwardly determined and a suc-

TABLE II. The parameters for the $p \bar{p}$ forward slope $B_{e l}(s)$ $\left(\chi^{2}=0.71018\right)$.

\begin{tabular}{cccc}
\hline \hline Process & $b_{R}(m b)$ & $R_{1}\left(m b^{2}\right)$ & $R_{2}\left(m b^{2}\right)$ \\
\hline$p \bar{p}$ & 4.62 & -99.7 & 22.39 \\
\hline \hline
\end{tabular}



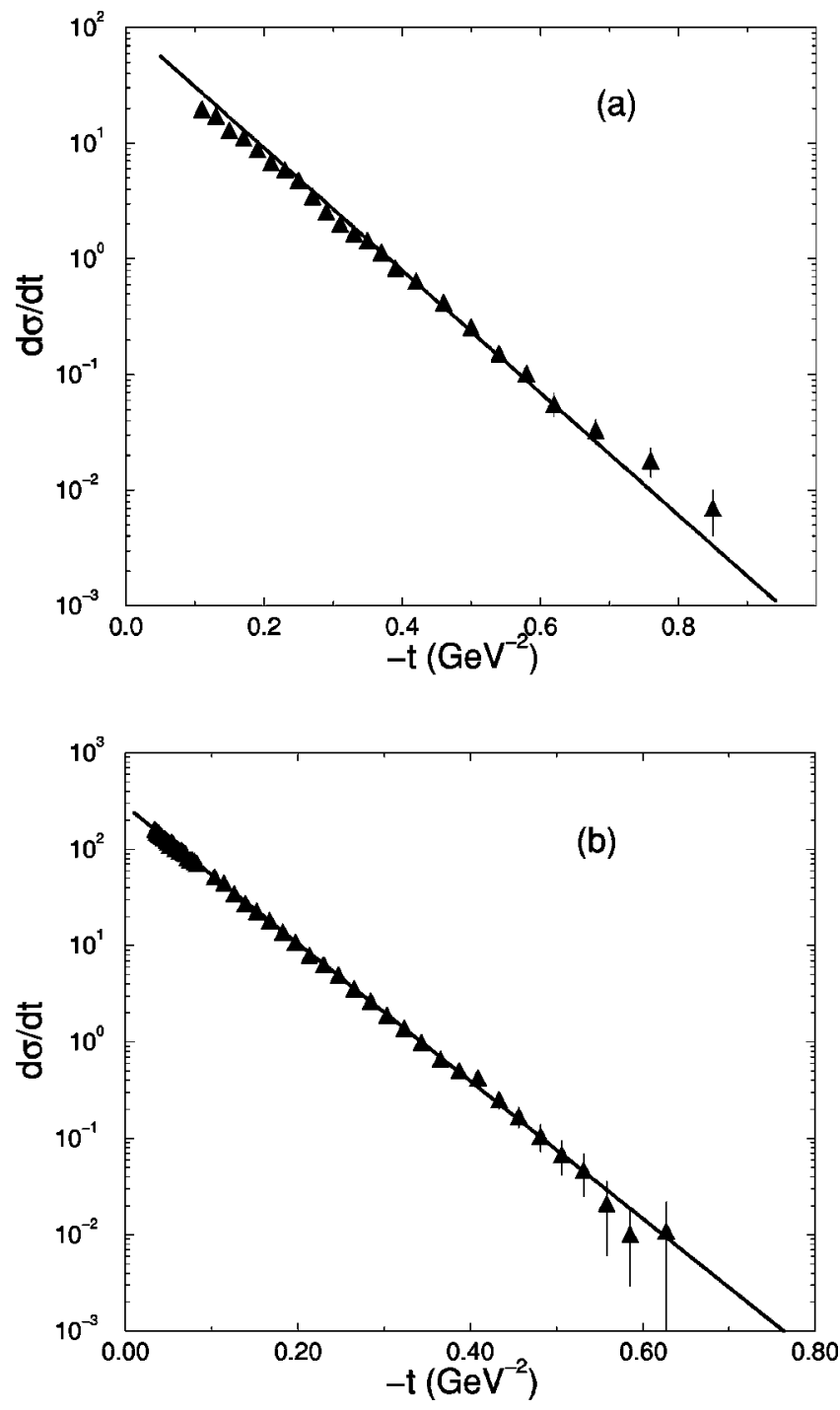

FIG. 6. The result for the elastic differential cross section at (a) $\sqrt{s}=53 \mathrm{GeV}$ and (b) $\sqrt{s}=1800 \mathrm{GeV}$, Ref. [15].

cessful comparison with its experimental measurements at $\sqrt{s}=53 \mathrm{GeV}$ and $\sqrt{s}=1800 \mathrm{GeV}$ is shown in Fig. 6 .

A reliable description of both total and differential cross sections is obtained, allowing the study of the role played by the impact factors in the calculations, for instance the factorizable feature of the Balitskiı and Kuchina impact factor.

It is well known that the large $t$ data are dominated by the perturbative contribution, as verified by DonnachieLandshoff in the calculation of three gluons exchange for the $p p(p \bar{p})$ reactions [23]. A further analysis will require the complete elastic amplitude rather than the small $t$ approximation, i.e., to describe the large $t$ region and extend the model to a wider interval in the momentum transfer. The $p p$ reaction presents the typical dips at momentum transfer of order $1-2 \mathrm{GeV}^{2}$ [23], which is not included in the small $t$ approximation. The usual procedure to solve this problem is by eikonalizing the Born amplitude, whose physical picture is the multiple elastic scattering of the Pomeron exchange [24]. In the present case, the Born amplitude does not violate the unitarity constraint and such a procedure seems not to be necessary. However, the dips structure can be present in the amplitude, i.e., the whole $t$ domain can be described taking a suitable choice of the impact factor.

\section{CONCLUSIONS}

We study in detail the contribution of a truncated BFKL series to the hadronic process, specifically the proton-proton (antiproton) collisions, considering two orders in perturbation theory corresponding to the two Reggeized gluons exchange and the one rung gluon ladder (considering the effective vertex). Despite the restrictions imposed by the use of a perturbative approach for soft observables, a good description of the total cross sections was obtained motivating an analysis of the elastic differential cross section. Although the QCD perturbation theory is in principle not reliable at the forward direction $(t=0)$, nevertheless we suppose that perturbation theory gives the behavior on energy even in this region. The next step is to consider $t$ different from zero, where the momentum transfer furnishes a scale to perform suitable calculations. In order to proceed this, we calculate the nonforward amplitude introducing two distinct Ansätze for the proton impact factor, namely, a factorizable $t$ dependent proposed recently by Balitskiı and Kuchina and the usual nonperturbative impact factor. In order to describe data we used a small momentum-transfer approximation and obtained an expression to the elastic slope $B_{e l}(s)$, determining the correspondent parameters. The elastic differential cross section is obtained straightforwardly, describing with good agreement the experimental data at both low- and highenergy values.

\section{ACKNOWLEDGMENTS}

This work was partially financed by $\mathrm{CNPq}$ and by PRONEX (Programa de Apoio a Núcleos de Excelência), Brazil.
[1] E. A. Kuraev, L. N. Lipatov, and V. S. Fadin, Phys. Lett. 60B, 50 (1975); Zh. Éksp. Teor. Fiz. 71, 840 (1976) [Sov. Phys. JETP 44, 443 (1976)]; 72, 377 (1977) [45, 199 (1977)]; Ya. Balitskiı̌ and L. N. Lipatov, Yad. Fiz. 28, 1597 (1978) [Sov. J. Nucl. Phys. 28, 822 (1978)].

[2] P. D. B. Collins, Introduction to Regge Theory and High Energy Physics (Cambridge University, Cambridge, England, 1977).
[3] M. Froissart, Phys. Rev. 123, 1053 (1961); A. Martin, ibid. 129, 1462 (1963).

[4] See, for instance, J. Bartels, Z. Phys. C 62, 425 (1994); J. Bartels and C. Ewerz, J. High Energy Phys. 09, 026 (1999); C. Ewerz, Phys. Lett. B 472, 135 (2000).

[5] Y. Kovchegov, Phys. Rev. D 61, 074018 (2000).

[6] R. Fiore, L. L. Jenkovszky, A. Lengyel, F. Paccanoni, and A. Papa, "The Pomeron as a finite sum of the gluon ladders," 
hep-ph/0002100.

[7] D. Kharzeev and E. Levin, Nucl. Phys. B578, 351 (2000).

[8] C. Augier et al., Phys. Lett. B 315, 503 (1993).

[9] V. S. Fadin and L. N. Lipatov, Phys. Lett. B 429, 127 (1998).

[10] V. S. Fadin and A. D. Martin, Phys. Rev. D 60, 114008 (1999).

[11] I. Balitskiı̌ and E. Kuchina, Phys. Rev. D 62, 074004 (2000).

[12] A. J. Askew, J. Kwieciński, A. D. Martin, and P. J. Sutton, Phys. Rev. D 49, 4402 (1994).

[13] J. R. Forshaw and D. A. Ross, QCD and the Pomeron (Cambridge University, Cambridge, England, 1997).

[14] V. S. Fadin and L. N. Lipatov, Phys. Lett. B 429, 127 (1998), and references therein; M. Ciafaloni, ibid. 429, 363 (1998); G. Camici and M. Ciafaloni, ibid. 430, 349 (1998); G. P. Salam, Acta Phys. Pol. B 30, 3679 (1999); M. Ciafaloni, D. Colferai, and G. P. Salam, Phys. Rev. D 60, 114036 (1999).

[15] The data used have been extracted from the PPDS acessible at http://pdg.lbl.gov (computer readable data files).
[16] A. Donnachie and P. V. Landshoff, Phys. Lett. B 296, 227 (1992).

[17] E710 Collaboration, S. Shukla et al., Nucl. Phys. B (Proc. Suppl.) 25B, 11 (1992).

[18] M. Honda et al., Phys. Rev. Lett. 70, 525 (1993).

[19] R. D. Field, Applications of Perturbative QCD (AddisonWesley, New York, 1989); F. Mandl and G. Shaw, Quantum Field Theory (Wiley, New York, 1988).

[20] F. Halzen, G. Krein, and A. A. Natale, Phys. Rev. D 47, 295 (1993); M. B. Gay Ducati, F. Halzen, and A. A. Natale, ibid. 48, 2324 (1993).

[21] J. R. Forshaw and P. J. Sutton, Eur. Phys. J. C 1, 285 (1998).

[22] G. Matthiae, Rep. Prog. Phys. 57, 743 (1994).

[23] A. Donnachie and P. V. Landshoff, Phys. Lett. 123B, 345 (1983); Nucl. Phys. B266, 960 (1986).

[24] See, for instance, M. Giffon, E. Martynov, and E. Predazzi, Z. Phys. C 76, 155 (1997); J. R. M. Covolan et al., ibid. 58, 109 (1993); E. Gotsman, E. M. Levin, and U. Maor, Phys. Lett. B 438, 229 (1998). 